\title{
Autism, Processing Speed, and Adaptive Functioning in Preschool Children
}

\author{
Åsa Hedvall, ${ }^{1,2}$ Elisabeth Fernell, ${ }^{1,3}$ Anette Holm, ${ }^{2}$ Jakob Åsberg Johnels, ${ }^{1,4}$ \\ Christopher Gillberg, ${ }^{1}$ and Eva Billstedt ${ }^{1}$ \\ ${ }^{1}$ Gillberg Neuropsychiatry Centre, Sahlgrenska Academy, University of Gothenburg, Kungsgatan 12, 411 19 Gothenburg, Sweden \\ ${ }^{2}$ Department of Psychology, Astrid Lindgren Children's Hospital, Stockholm, 17176 Stockholm, Sweden \\ ${ }^{3}$ Skaraborgs's Hospital, Department of Pediatrics, Research and Development Center and Unit of Developmental Disorders, \\ Skaraborg's Hospital, 54185 Skövde, Sweden \\ ${ }^{4}$ Department of Psychology, University of Gothenburg, Box 500, 40530 Gothenburg, Sweden
}

Correspondence should be addressed to Åsa Hedvall; asa.lundholm-hedvall@karolinska.se

Received 25 February 2013; Accepted 16 April 2013

Academic Editors: R. J. Beninger, S. A. Freedman, and R. R. Tampi

Copyright ( 2013 Åsa Hedvall et al. This is an open access article distributed under the Creative Commons Attribution License, which permits unrestricted use, distribution, and reproduction in any medium, provided the original work is properly cited.

\begin{abstract}
Objectives. To study cognitive test profiles with a focus on processing speed in a representative group of preschool children with autism spectrum disorder (ASD) and relate processing speed to adaptive functioning. Methods. Cognitive assessments were performed in 190 3.6-6.6-year-old children (164 boys and 26 girls) with ASD, using either Griffiths' developmental scales $(n=77)$ or the Wechsler Preschool and Primary Scale of Intelligence-Third Edition (WPPSI-III) $(n=113)$. Cognitive data were related to adaptive functioning as measured by Vineland Adaptive Behavior Scales (VABS). Results. Cognitive profiles were characterized by low verbal skills. Low processing speed quotients (PSQs) were found in $66(78 \%)$ of the 85 children who were able to participate in the processing speed subtests. Except for Socialization, all VABS domains (Communication, Motor Skills, Daily Living Skills, and Adaptive Behavior Composite scores) correlated significantly with PSQ. Multiple regression analysis showed that PSQ predicted 38\%, 35\%, 34\%, and 37\% of the variance for Communication, Daily Living Skills, Motor Skills, and total Adaptive Composite scores, respectively. Conclusion. Preschool children with ASD had uneven cognitive profiles with low verbal skills, and, relatively, even lower PSQs. Except for Socialization, adaptive functioning was predicted to a considerable degree by PSQ.
\end{abstract}

\section{Introduction}

Autism spectrum disorders (ASDs) are common disabling conditions with a heterogeneous etiology and clinical presentation as well as a high degree of overlap with other neurodevelopmental disorders.

Diagnosing ASD in young children can be difficult due to the coexistence and overlap with many other developmental disorders, such as developmental coordination disorder, attention-deficit/hyperactivity disorder, speech and language disorders, and/or general developmental delay/impairment, that is, other ESSENCE conditions $[1,2]$. Moreover, the full clinical picture often changes during early preschool years and may not have fully appeared at the age of 2-3 years, an age window when different child health screening programs are in use $[3,4]$. ASD is linked to a variety of cognitive difficulties affecting the individual and with implications for the child's interaction with his or her family, peers, and in the preschool/school setting.

Intellectual disability (ID) is one of the most common co-occurring disorders in ASD $[5,6]$ and is an important predictor of outcome [7-10]. In a recent study of preschool children with ASD (Hedvall et al., 2013, submitted), we have reported that a test result in the broad range of Intellectual Disability Developmental Quotient (DQ)/Intellectual Quotient (IQ) $<70$ at initial preschool assessment was stable at reassessment two years later, whereas a borderline result (IQ/DQ $=70-84)$ was just as likely to go down, up, or stay in the same range at followup.

Many studies have demonstrated that an uneven profile is characteristic of individuals with ASD with general relative strengths in visual-spatial nonverbal measures and a 
concurrent weakness in verbal ability [11-16]. Except for the impact of general intelligence on outcome of ASD, no single cognitive model provides a full explanation of the multiplicity of the clinical presentations in ASD. Two well-known theories are the Theory of Mind hypothesis and the Central Coherence Theory. Theory of Mind is the ability to attribute mental states to self and others and to understand that others have beliefs, desires, and intentions that are different from one's own [17]. Weak central coherence refers to the detailed "peripheral" focused processing style, characteristic of ASD, whereas typically developing children and adults tend to process incoming information for meaning and global understanding [18].

There has also been a growing research interest regarding different executive functions; including planning, working memory, impulse control, and shifting set in individuals with ASD. In a review, Russo and collaborators [19] discussed the importance of understanding executive function in ASD and its relation to general developmental level and in impairments of set shifting/mental flexibility in older groups of children. An early study of executive dysfunction and mental flexibility in preschool children with ASD was conducted by McEvoy et al. [20]. Children with ASD (mean age 5 years) were found to have selective deficits in executive function compared to both developmental delayed children of similar nonverbal mental age and to normally developing children. Pellicano [21] studied the link between theory of mind and executive function in young children with autism and in typically developing children. A significant correlation emerged between theory of mind and executive variables in the autism group. Executive dysfunction may also explain the repetitive behaviors and restricted interests seen in individuals with ASD [22]. Particularly, executive dysfunctions regarding attention, set shifting, and planning have been reported in young children with ASD [23]. Poor mental flexibility is considered to give a more rigid and concrete bound behavior, occasionally transformed into perseverations [24].

Processing speed may be analogous to the operating speed of the central processing unit of a computer [25] and is related to performance of higher-order cognition. Processing speed was found to be weakest relative to other indices using Wechsler Intelligence Scale for Children-Fourth Edition (WISC-IV) in a group of high-functioning (IQ > 70) children (age 10) with autism [26]. Slow processing speed may give problems with rate of learning, comprehension of new information, and mental fatigue. The study by Mayes and Calhoun [27] indicated that learning, attention, graphomotor and processing speed deficits tended to go together in children with ADHD and high-functioning autism compared to other clinical disorders. Processing speed deficits have been found in hypoactive and inattentive children with Attention Deficit Hyperactivity Disorder (ADHD), using the WISCIV $[28,29]$, but have also been demonstrated in the ADHD group in general [30].

Processing speed deficits can be expected to influence many daily activities with demands on completing tasks and responsibilities on time and thus being a cognitive factor of great importance in everyday life. The deficits are in many ways "invisible," and children with ASD and normal IQ, but with processing speed difficulties, may, if this problem is not recognized, fail to carry out tasks that are expected of them without this being an effect of the ASD "per se." Whereas several research studies exist that have established the impact of general IQ on adaptive (daily life) functions in ASD samples [31, 32], little research has examined which aspect of a child's IQ it is that has the greatest role in this respect. We have not been able to locate any ASD-related studies that specifically look at associations between processing speed and adaptive functioning across several adaptive domains, except for the association between processing speed and communication, indicating speed to correlate positively with communication abilities [26].

The aim of the present study, therefore, was to analyze developmental and cognitive test data (Griffiths' and WPPSI) $[33,34]$ in a representative group of preschool children with a clinical diagnosis of ASD, particularly with regards to processing speed, and to relate this result to aspects of daily life functioning, as measured by the VABS [35].

\section{Methods}

2.1. Procedure. The sample was drawn from a community representative group of 208 children with clinically diagnosed ASD in the county of Stockholm, previously described in detail by Fernell et al. [6, 36]. The children had had indepth assessments prior to and at referral to a specialized habilitation center, the Autism Center for Young Children (ACYC), and at a follow-up at the center after 2 years. Of the 208 children, referred to the ACYC, 198 participated in the 2year follow-up, and of these, 196 participated in a cognitive assessment. The majority, 190 children, were assessed by either of the two research psychologists at the ACYC and therefore included in this study. The remaining 6 children were reassessed by referral team psychologists.

2.2. Participants. The 190 children-26 (14\%) girls and 164 (86\%) boys-were aged between 3.6 and 6.6 years (mean age $5.5,(\mathrm{SD}=0.8)$ at the time of the study. The distribution of ASD subtypes at the 2-year follow-up, using the DSM-IV criteria (APA, 1994) [37], was 100 (53\%) children with autistic disorder ( 87 boys and 13 girls), 56 (30\%) with Pervasive Developmental Disorder-Not Otherwise Specified (PDDNOS) (50 boys and 6 girls), and 12 (6\%) with Asperger syndrome (10 boys and 2 girls). A subgroup of 21 children (11\%, 16 boys and 5 girls) had some autistic symptoms but not enough to meet criteria for a full ASD diagnosis at followup. One child did not have an assessment with regard to ASD subtype.

Of the 190 children, a total of 87 (46\%) had ID, 51 (27\%) borderline IQ/DQ, and 52 (27\%) an average IQ/DQ [36].

Of the 18 children who did not participate in the cognitive follow-up assessment presented here, 12 children had autistic disorder at referral to the ACYC ( 8 with ID and 4 with borderline IQ/DQ) and 6 children had atypical autism ( 2 with average IQ/DQ and 4 with borderline IQ/DQ). 


\subsection{Developmental-Cognitive Tests}

2.3.1. Griffiths' Developmental Scales I and/or II. For children with a mental age $<2.6$ years, intelligence/mental age was assessed with the Swedish versions of Griffiths' Developmental Scales [33]. Developmental quotients (DQs) for the total and subscale scores obtained were converted to IQ equivalents in order to obtain a score corresponding to intelligencequotient points. Results from Griffiths' scale C (Hearing and Speech) and, when available, scale F (Practical Reasoning, for children with mental age $>24$ months) were converted to Verbal Function, and scale D (Eye and Hand Coordination) and scale E (Performance) were converted to Performance Function. Global Cognitive Function is the average value of verbal function and performance function [38].

2.3.2. Wechsler Preschool and Primary Scale of IntelligenceThird Edition (WPPSI-III) [34]. WPPSI-III [34] was used for children with a mental age $>2.6$ years, providing full scale IQ (FSIQ), verbal IQ (VIQ), and performance IQ (PIQ). WPPSI-III generates also Processing Speed Quotient (PSQ) and General Language Composite (GLC). VIQ, PIQ, and FSIQ are referred to as Verbal, Performance, and Global Cognitive Function, respectively.

WPPSI-III version for age span 2.6-3.11 years includes 4 core subtests (Receptive Vocabulary, Information, Block Design, and Object Assembly) and 1 supplemental subtest (Picture Naming). Four composite scores are possible for this age band: VIQ, PIQ, FSIQ, and GLC.

WPPSI-III version for age span 4.0-7.3 includes 7 core subtests (Information, Vocabulary, Word Reasoning, Block Design, Matrix Reasoning, Picture Concepts, and Coding) and 5 supplemental subtest (Similarities, Comprehension, Object Assembly, Picture Completion, and Symbol Search). A total of five composite scores are possible for this age band: VIQ, PIQ, PSQ, FSIQ, and GLC. Receptive Vocabulary and Picture Naming are included in the GLC and Symbol Search and Coding in the PSQ.

\subsection{Adaptive Functioning Scale}

2.4.1. Vineland Adaptive Behavior Scales (VABS-II). The VABS-II [35] is an informant-based measure of adaptive behavior that yields a composite score and four domain scores: Communication (receptive, expressive, and written adaptive functions), Daily Living Skills (personal, domestic, and community skills), Socialization (interpersonal relationships, play and leisure time, and coping abilities), and Motor skills (gross and fine motor skills).

2.5. Statistical Methods. Statistical analyses were carried out using SPSS version 19 (SPSS, Chicago, IL, USA). Pearson's $r$ was used to investigate the relationship between PSQ and VABS. Those variables that were significantly correlated with the criterion variable (VABS-II domains) were entered as predictors into a multiple regression model using the standard (enter) method. An alpha level of .05 was used for all statistical analyses.

\section{Results}

3.1. Cognitive and Developmental Test Data. Intellectual/developmental levels and profiles according to WPPSI-III and the Griffiths' test are presented in Table 1.

Seventy-seven of the 190 children (40\%) were assessed with Griffiths' Developmental Scales. For those who were evaluated using Griffiths' developmental scales, the mean verbal function was 33.5 ( $S D=15.9)$, performance function was $46.7(\mathrm{SD}=16.3)$, and global function was $41.6(\mathrm{SD}=14.5)$ (Table 1).

One hundred and thirteen children $(60 \%$ of the total sample) were tested with the WPPSI-III, 112 with the version for age span 4.0-7.3 years, and one child with the version for age span 2.6-3.11 years. FSIQ for this group was 84.5 (SD = 14.7), VIQ $84.8(\mathrm{SD}=16.1)$, and PIQ $93.6(\mathrm{SD}=16.7)$. Mean value for PSQ $(n=85)$ was $76.7(\mathrm{SD}=12.2)$ and for GLC $(n=99)$ was $89.9(\mathrm{SD}=17.1)$. The differences between PSQ, VIQ, PIQ, and GLC were significant $(P<.000)$.

On a group level, the cognitive profile was uneven in both Griffiths' and WPPSI-III with a significantly lower verbal function compared to performance (Griffiths' $P=.01$; WPPSI-III; $P=.01$ ). No significant difference was found between boys and girls.

3.2. PSQ. Twenty-five of 113 children (22\%) who were tested with WPPSI-III were not able to participate in the subtests Coding and Symbol Search that together comprise the PSQ. FSIQ in this "non- PSQ" group was $76.3(\mathrm{SD}=14.9)$ compared to a mean FSIQ of $86.8(\mathrm{SD}=13.8, P=.003)$ in the group who were able to perform PSQ subtests. There was also a difference regarding PIQ in the two groups, and mean PIQ was 83.5 $(\mathrm{SD}=17.8)$ in the "non-PSQ" group compared to 96.5 (SD $=15.3, P=.002)$ in the PSQ-group. No difference was found regarding VIQ $(M=79.6, \mathrm{SD}=17.4$ and $M=86.3, \mathrm{SD}=15.4)$ between the two groups.

PSQ in relation to VABS-II was available for 84 of the 190 children. There were significant positive correlations between PSQ and the Communication domain $(r=.422, P=.01)$, Motor Skills domain $(r=.414, P=.01)$, Daily Living Skills (DLS) domain $(r=.377, P=.01)$, and Adaptive Composite score $(r=.438, P=.01)$ (Table 2$)$.

3.2.1. PSQ and Statistical Predictions of VABS. To examine the role of PSQ as a unique statistical predictor of adaptive functioning, we next performed multiple regression analyses. Four separate analyses were performed using the three VABS subdomain scores that were significantly related to PSQ (i.e., all but socialization) and the total composite score as the dependent variables. PSQ, VIQ, and PIQ were entered simultaneously as independent variables. PSQ predicted $38 \%$ of the communication ( $\beta=.301, P=.005)$ scale, $35 \%$ of variance for DLS $(\beta=.354, P=.004), 34 \%$ for the motor skills domain $(\beta=.341, P=.004)$, and $37 \%$ for the total composite score $(\beta=.373, P=.001)$. VIQ predicted $24 \%$ of motor skills domains $(\beta=.241, P=.049)$ and $28 \%$ of total composite score $(\beta=.277, P=.022)$, whereas PIQ did not predict any of the VABS scores (all Ps $>.66$ ). 
TABLE 1: Cognitive function measured by WPPSI-III index and subtests results and by Griffiths' Developmental Scales, Adaptive behavior measured by Vineland Adaptive Behavior Scales.

\begin{tabular}{|c|c|c|c|c|c|}
\hline \multirow{2}{*}{ Index/subtests/development scales } & \multicolumn{5}{|c|}{ WPPSI-III } \\
\hline & $N$ & $\mathrm{IQ} / \mathrm{DQ} /$ scores & Subtest mean & Std. deviation & Range \\
\hline WPPSI verbal IQ (VIQ) & 113 & 84,8 & & 16,1 & $53-133$ \\
\hline Information & 112 & & 7,4 & 3,3 & $1-16$ \\
\hline Vocabulary & 110 & & 7,4 & 2,9 & $2-16$ \\
\hline Word Reasoning & 111 & & 7,2 & 3,1 & $1-15$ \\
\hline Comprehension & 63 & & 9,0 & 2,9 & $3-15$ \\
\hline Similarities & 67 & & 7,9 & 2,6 & $3-15$ \\
\hline WPPSI performance IQ (PIQ) & 113 & 93,6 & & 16,7 & $53-135$ \\
\hline Block Design & 112 & & 10,3 & 3,6 & $1-18$ \\
\hline Matrix Reasoning & 111 & & 8,8 & 3,5 & $1-16$ \\
\hline Picture Concepts & 111 & & 8,2 & 3,4 & $1-17$ \\
\hline Picture Completion & 67 & & 9,6 & 3,5 & $2-19$ \\
\hline WPPSI Processing speed (PSQ) & 85 & 76,7 & & 12,2 & $49-107$ \\
\hline Coding & 110 & & 5,1 & 2,9 & $1-12$ \\
\hline Symbol Search & 95 & & 5,9 & 2,6 & $2-14$ \\
\hline WPPSI General Language (GLC) & 99 & 89,9 & & 17,1 & $45-128$ \\
\hline Receptive Vocabulary & 96 & & 8,2 & 3,1 & $1-15$ \\
\hline Picture Naming & 95 & & 8,5 & 3,2 & $2-16$ \\
\hline WPPSI full scale IQ (FSIQ) & 113 & 84,5 & & 14,7 & $51-121$ \\
\hline Griffiths' verbal functioning & 77 & 33,5 & & 15,9 & $8-72$ \\
\hline Griffiths' performance functioning & 77 & 46,7 & & 16,3 & $15-79$ \\
\hline Griffiths' global functioning & 77 & 41,6 & & 14,5 & $13-73$ \\
\hline VABS Communication & 187 & 71,9 & & 18,9 & $34-120$ \\
\hline VABS DLS & 187 & 73,8 & & 16,4 & $38-117$ \\
\hline VABS Socialization & 187 & 70,6 & & 14,7 & $46-112$ \\
\hline VABS Motor & 187 & 76,4 & & 14,5 & $43-117$ \\
\hline VABS total & 187 & 70,5 & & 15,0 & $42-114$ \\
\hline
\end{tabular}

WPPSI-III index scale and Griffiths' scales mean of 100 and a standard deviation of 15, WPPSI-III subtest scale point range 1-19, mean 10.

VABS: Vineland Adaptive Behavior Scales.

DLS: Daily Living Skills.

IQ: Intellectual Quotient.

DQ: Developmental Quotient.

\section{Discussion}

In accordance with results obtained in previously published studies, the cognitive profiles in our group of preschool children with ASD were characterized by significantly higher performance than verbal skills. This profile was evident both in the higher functioning group tested with WPPSI-III and in those with lower functioning, tested with Griffiths' developmental scales.

Results on the PSQ were significantly depressed compared to both verbal and performance IQ scores. Moreover, results on the two subtests composing PSQ, Coding, and Symbol search were equally low, indicating that these two subtests require cognitive efforts and that the motor function per se is not decisive for the Coding subtest. Analysis of the group that did not accomplish the subtests of the PSQ revealed that this group had a lower mean FSIQ. This finding supports the requirement for cognitive abilities in order to complete the Coding and Symbol search subtests, and, hence, our "PSQ group" can be regarded as a more able ASD group. Processing Speed subtests challenge the child's capacity to work independently according to a given template, and they require graphomotor speed, accuracy, and mental flexibility/set shifting capacity in order to sustain attention to task. Processing speed may be especially important in assessing young children due to its relationship to other cognitive abilities and learning. Clinical developmental research suggests a dynamic interplay between working memory, processing speed, and reasoning. More rapid processing of information enhances the effectiveness of working memory which enhances reasoning ability. Children with processing speed problems might therefore have more difficulties with task that requires working memory and reasoning ability, which both are needed in acquisition of new information [39]. These abilities reflect important executive functions, and our findings, hence, indicate that executive functions may be impaired already at early preschool age. However, demands on executive functions during preschool age are limited, 
TABLE 2: Pearson correlations between WPPSI-III index scores and Vineland Adaptive Behavior Scales Domains.

\begin{tabular}{|c|c|c|c|c|c|c|c|c|c|c|}
\hline & \multicolumn{5}{|c|}{ WPPSI-III } & \multicolumn{5}{|c|}{ Vineland Adaptive Behavior Scales Domains } \\
\hline & VIQ & PIQ & PSQ & FSIQ & GLC & Communication & DLS & Socialization & Motoric & Composite \\
\hline VIQ & 1 &, $529^{* *}$ &, $373^{* *}$ & $869^{* *}$ &, $810^{* *}$ &, $538^{* *}$ & $223^{*}$ & $227^{*}$ & $312^{* *}$ & $376^{* *}$ \\
\hline PIQ & & 1 & $475^{* *}$ &, $854^{* *}$ &, $435^{* *}$ & $409^{* *}$ &, $322^{* *}$ &, $237^{*}$ &, $359^{* *}$ &, $389^{* *}$ \\
\hline PSQ & & & 1 &, $585^{* *}$ &, $278^{*}$ &, $422^{* *}$ &, $377^{* *}$ & ,206 &, $414^{* *}$ &, $438^{* *}$ \\
\hline FSIQ & & & & 1 &, $713^{* *}$ &, $557^{* *}$ &, $322^{* *}$ &, $251^{* *}$ &, $403^{* *}$ &, $451^{* *}$ \\
\hline GLC & & & & & 1 &, $462^{* *}$ &, $210^{*}$ &, $231^{*}$ &, $381^{* *}$ &, $366^{* *}$ \\
\hline Communication & & & & & & 1 &, $832^{* *}$ &, $769^{* *}$ &, $751^{* *}$ &, $921^{* *}$ \\
\hline DLS & & & & & & & 1 &, $822^{* *}$ &, $802^{* *}$ &, $939^{* *}$ \\
\hline Socialization & & & & & & & & 1 &, $712^{* *}$ &, $900^{* *}$ \\
\hline Motoric & & & & & & & & & 1 &, $890^{* *}$ \\
\hline
\end{tabular}

${ }^{* *}$ Correlation is significant at the 0.01 level (2-tailed).

* Correlation is significant at the 0.05 level (2-tailed).

VIQ: Verbal IQ.

PIQ: Performance IQ.

PSQ: Processing Speed Quotient.

FSIQ: Full Scale IQ.

GLC: Global Language Composite.

DLS: Daily Living Skills.

and requirements for these abilities will increase significantly when the child starts school.

Salcedo-Marin et al. [40] studied the executive problems of school-aged children with either ASD or ADHD, especially with regards to planning ability. The authors discussed that despite overlapping clinical and cognitive features between the two disorders, children with ASD and with ADHD presented a different pattern in planning function performance. In children with ASD, planning function problems seemed to be mediated by processing speed and motor coordination and not by other executive function problems, including attention, working memory, or response inhibition. Clinical and educational implications of the findings were also discussed.

Our finding that PSQ was significantly correlated to adaptive functions also indicated that processing speed ability will affect everyday functioning. Similar results have been found in a study of school-age children with ASD [26] reporting that processing speed was the greatest area of weakness in the ASD group. The authors found that more than half of the sample scored at least a full standard deviation below the processing speed normative mean score and that processing speed performance also related to autism communication symptoms and adaptive communication abilities.

Our study group had not started school at the time of assessment, but it would probably be of importance that the low PSQ and ensuing implications for school work are conveyed to the child's school teachers. The mediating effect of cognitive processing speed on the ability to achieve the full potential of intellectual functioning at school, that is, the importance of detecting "slow" children, was emphasized by Lundervold and collaborators [29]. In cognitively gifted students with ASD, working memory and processing speed indices were both significantly positively correlated with achievement in math, reading, and written language [41], thus also highlighting the importance of paying attention to this cognitive factor at school.

In our study group, about half the children had ID [36], and they will therefore have the right to receive adapted education in the special schools. However, the rate of ID will decrease in a group of children with ASD at school age when also milder forms of ASD, without ID, have been identified and diagnosed. Among school age children with ASD, the rate of ID can be estimated to be $15 \%-20 \%$ [2]. In our opinion, processing speed results, as markers and predictors for executive and adaptive functions, should be more highlighted in the teaching situation for children with ASD.

Processing speed deficits are common in many ESSENCE conditions, and this underscores the cognitive overlap across many of these disorders and the need for a broad perspective when assessing children with different kinds of developmental disorders/problems.

We have captured cognitive profiles at a specific time point at preschool age, and we can expect that development/maturation may change in some children over time. A limitation of the study was that more than half the group could not participate in the PSQ subtests. The reasons for that were that the WPPSI test could not be used at all in one group and there was also a group that could not participate in the PSQ subtests, although they could complete the other WPPSI subtests. Thus, our results are valid for the more able ASD group. In future research, there is a need for cognitive follow-up studies to examine stability of cognitive profiles and adaptive outcome.

\section{Ethical Approval}

The study was approved by the Ethics Committee in Stockholm. 


\section{Acknowledgments}

The authors are very grateful to all parents and children participating in the study and to personnel at the Autism Centre for Young Children. The project was financially supported by the Gillberg Neuropsychiatry Centre, the Wilhelm and Martina Lundgren Foundation, and the Department of Psychology at Karolinska University Hospital.

\section{References}

[1] T. Charman and G. Baird, "Practitioner review: diagnosis of autism spectrum disorder in 2- and 3-year-old children," Journal of Child Psychology and Psychiatry and Allied Disciplines, vol. 43, no. 3, pp. 289-305, 2002.

[2] C. Gillberg, "The ESSENCE in child psychiatry: early symptomatic syndromes eliciting neurodevelopmental clinical examinations," Research in Developmental Disabilities, vol. 31, no. 6, pp. 1543-1551, 2010.

[3] T. Charman, E. Taylor, A. Drew, H. Cockerill, J. A. Brown, and G. Baird, "Outcome at 7 years of children diagnosed with autism at age 2: predictive validity of assessments conducted at 2 and 3 years of age and pattern of symptom change over time," Journal of Child Psychology and Psychiatry and Allied Disciplines, vol. 46, no. 5, pp. 500-513, 2005.

[4] G. Nygren, E. Sandberg, F. Gillstedt, G. Ekeroth, T. Arvidsson, and C. Gillberg, "A new screening programme for autism in a general population of Swedish toddlers," Research in Developmental Disabilities, vol. 33, no. 4, pp. 1200-1210, 2012.

[5] J. L. Matson and M. Shoemaker, "Intellectual disability and its relationship to autism spectrum disorders," Research in Developmental Disabilities, vol. 30, no. 6, pp. 1107-1114, 2009.

[6] E. Fernell, A. Hedvall, F. Norrelgen et al., "Developmental profiles in preschool children with autism spectrum disorders referred for intervention," Research in Developmental Disabilities, vol. 31, no. 3, pp. 790-799, 2010.

[7] K. S. Wallace and S. J. Rogers, "Intervening in infancy: implications for autism spectrum disorders," Journal of Child Psychology and Psychiatry and Allied Disciplines, vol. 51, no. 12, pp. 1300-1320, 2010.

[8] P. Howlin, S. Goode, J. Hutton, and M. Rutter, "Adult outcome for children with autism," Journal of Child Psychology and Psychiatry and Allied Disciplines, vol. 45, no. 2, pp. 212-229, 2004.

[9] C. Fountain, A. S. Winter, and P. S. Bearman, "Six developmental trajectories characterize children with autism," Pediatrics, vol. 129, no. 5, pp. el112-e1120, 2012.

[10] E. Billstedt, C. Gillberg, and C. Gillberg, "Autism after adolescence: population-based 13- to 22-year follow-up study of 120 individuals with autism diagnosed in childhood," Journal of Autism and Developmental Disorders, vol. 35, no. 3, pp. 351-360, 2005.

[11] J. A. Burack, G. Iarocci, D. Bowler, and L. Mottron, "Benefits and pitfalls in the merging of disciplines: the example of developmental psychopathology and the study of persons with autism," Development and Psychopathology, vol. 14, no. 2, pp. 225-237, 2002.

[12] F. G. E. Happe, "Wechsler IQ profile and theory of mind in autism: a research note," Journal of Child Psychology and Psychiatry and Allied Disciplines, vol. 35, no. 8, pp. 1461-1471, 1994.
[13] R. M. Joseph, H. Tager-Flusberg, and C. Lord, "Cognitive profiles and social-communicative functioning in children with autism spectrum disorder," Journal of Child Psychology and Psychiatry and Allied Disciplines, vol. 43, no. 6, pp. 807-821, 2002.

[14] A. M. Girardot, S. De Martino, C. Chatel, D. Da Fonseca, V. Rey, and F. Poinso, "Cognitive profiles in pervasive developmental disorders," Encephale, vol. 38, no. 6, pp. 488-495, 2012.

[15] A. Shah and U. Fritht, "Why do autistic individuals show superior performance on the block design task?" Journal of Child Psychology and Psychiatry and Allied Disciplines, vol. 34, no. 8, pp. 1351-1364, 1993.

[16] A. D. Sandberg, A. Nydén, C. Gillberg, and E. Hjelmquist, “The cognitive profile in infantile autism - a study of 70 children and adolescents using the Griffiths Mental Development Scale," The British Journal of Psychology, vol. 84, p. 3, 1993.

[17] D. Premack and G. Woodruff, "Chimpanzee problem-solving: a test for comprehension," Science, vol. 202, no. 4367, pp. 532-535, 1978.

[18] U. Frith, "Cognitive explanations of autism," Acta Paediatrica, vol. 85, no. 416, pp. 63-68, 1996.

[19] N. Russo, T. Flanagan, G. Iarocci, D. Berringer, P. D. Zelazo, and J. A. Burack, "Deconstructing executive deficits among persons with autism: implications for cognitive neuroscience," Brain and Cognition, vol. 65, no. 1, pp. 77-86, 2007.

[20] R. E. McEvoy, S. J. Rogers, and B. F. Pennington, "Executive function and social communication deficits in young autistic children," Journal of Child Psychology and Psychiatry and Allied Disciplines, vol. 34, no. 4, pp. 563-578, 1993.

[21] E. Pellicano, "Links between theory of mind and executive function in young children with autism: clues to developmental primacy," Developmental Psychology, vol. 43, no. 4, pp. 974-990, 2007.

[22] E. L. Hill, "Executive dysfunction in autism," Trends in Cognitive Sciences, vol. 8, no. 1, pp. 26-32, 2004.

[23] C. Hughes, J. Russell, and T. W. Robbins, "Evidence for executive dysfunction in autism," Neuropsychologia, vol. 32, no. 4, pp. 477492, 1994.

[24] S. Ozonoff and J. Jensen, "Specific executive function profiles in three neurodevelopmental disorders," Journal of Autism and Developmental Disorders, vol. 29, no. 2, pp. 171-177, 1999.

[25] R. Kail and T. A. Salthouse, "Processing speed as a mental capacity," Acta Psychologica, vol. 86, no. 2-3, pp. 199-225, 1994.

[26] R. E. Oliveras-Rentas, L. Kenworthy, R. B. Roberson, A. Martin, and G. L. Wallace, "WISC-IV profile in high-functioning autism spectrum disorders: impaired processing speed is associated with increased autism communication symptoms and decreased adaptive communication abilities," Journal of Autism and Developmental Disorders, vol. 42, no. 5, pp. 655-664, 2012.

[27] S. D. Mayes and S. L. Calhoun, "Learning, attention, writing, and processing speed in typical children and children with ADHD, autism, anxiety, depression, and oppositional-defiant disorder," Child Neuropsychology, vol. 13, no. 6, pp. 469-493, 2007.

[28] S. D. Mayes and S. L. Calhoun, "WISC-IV and WISC-III profiles in children with ADHD," Journal of Attention Disorders, vol. 9, no. 3, pp. 486-493, 2006.

[29] A. J. Lundervold, M. B. Posserud, A. K. Ullebø, L. Sørensen, and C. Gillberg, "Teacher reports of hypoactivity symptoms reflect slow cognitive processing speed in primary school children," European Child and Adolescent Psychiatry, vol. 20, no. 3, pp. 121126, 2011. 
[30] N. Chhabildas, B. F. Pennington, and E. G. Willcutt, "A comparison of the neuropsychological profiles of the DSM-IV subtypes of ADHD," Journal of Abnormal Child Psychology, vol. 29, no. 6, pp. 529-540, 2001

[31] J. Schatz and G. Hamdan-Allen, "Effects of age and IQ on adaptive behavior domains for children with autism," Journal of Autism and Developmental Disorders, vol. 25, no. 1, pp. 51-60, 1995.

[32] A. Perry, H. E. Flanagan, J. Dunn Geier, and N. L. Freeman, "Brief report: the vineland adaptive behavior scales in young children with autism spectrum disorders at different cognitive levels," Journal of Autism and Developmental Disorders, vol. 39, no. 7, pp. 1066-1078, 2009.

[33] B. Alin-Åkerman and L. Nordberg, Griffiths'Developmental Scales I and II, Psykologiförlaget AB, Stockholm, Sweden, 1991.

[34] D. Wechsler, WPPSI-III. Wechsler Preschool and Primary Scale of Intelligence (Swedish Version), Psykologiförlaget AB, Stockholm, Sweden, 2005.

[35] S. S. Sparrow, D. V. Cicchetti, and D. A. Balla, Vineland Adaptive Behavior Scales, AGS, Circle Pines, Minn, USA, 2nd edition, 2005.

[36] E. Fernell, Å. Hedvall, J. Westerlund et al., "Early intervention in 208 Swedish preschoolers with autism spectrum disorder. A prospective naturalistic study," Research in Developmental Disabilities, vol. 32, no. 6, pp. 2092-2101, 2011.

[37] American Psychiatric Association (APA), Diagnostic and Statistical Manual of Mental Disorders (DSM-IV), American Psychiatric Association, Washington, DC, USA, 4th edition, 1994.

[38] M. L. Engman, I. Adolfsson, I. Lewensohn-Fuchs, M. Forsgren, M. Mosskin, and G. Malm, "Neuropsychologic outcomes in children with neonatal herpes encephalitis," Pediatric Neurology, vol. 38, no. 6, pp. 398-405, 2008.

[39] G. Goldstein and S. R. Beers, Comprehensive Handbook of Psychological Assessment, Intellectual and Neuropsychological Assessment, vol. 1, Jon Wiley and Sons, New Jersey, NJ, USA, 2004.

[40] M. D. Salcedo-Marin, J. M. Moreno-Granados, M. RuizVeguilla, and M. Ferrin, "Evaluation of planning dysfunction in attention deficit hyperactivity disorder and autistic spectrum disorders using the zoo map task," Child Psychiatry \& Human Development, vol. 44, no. 1, pp. 166-185, 2013.

[41] S. G. Assouline, M. Foley Nicpon, and L. Dockery, "Predicting the academic achievement of gifted students with autism spectrum disorder," Journal of Autism and Developmental Disorders, vol. 42, no. 9, pp. 1781-1789, 2012. 


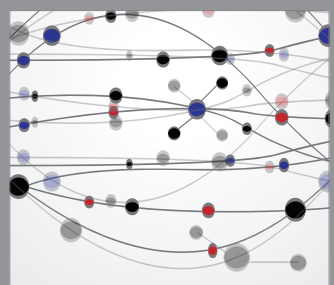

The Scientific World Journal
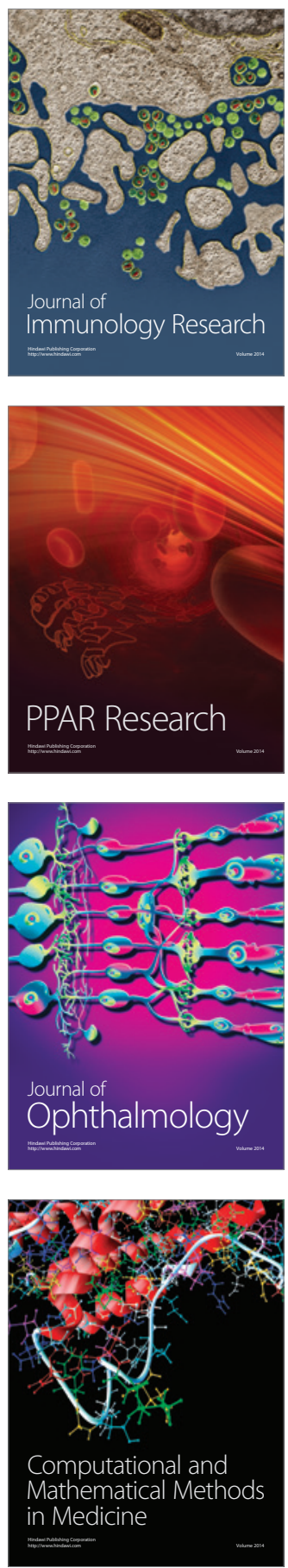

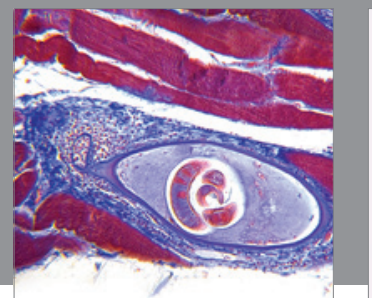

Gastroenterology

Research and Practice
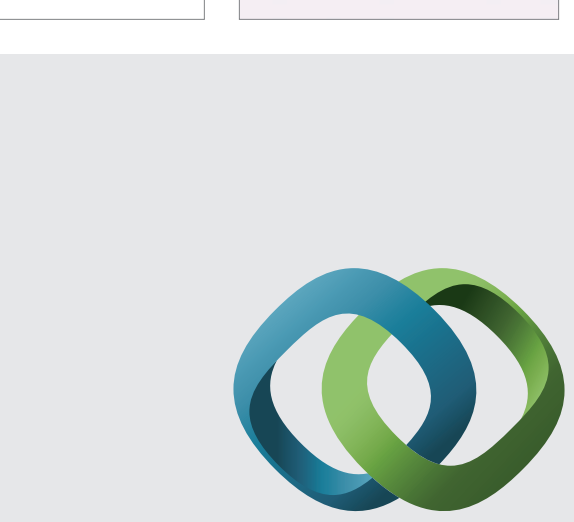

\section{Hindawi}

Submit your manuscripts at

http://www.hindawi.com
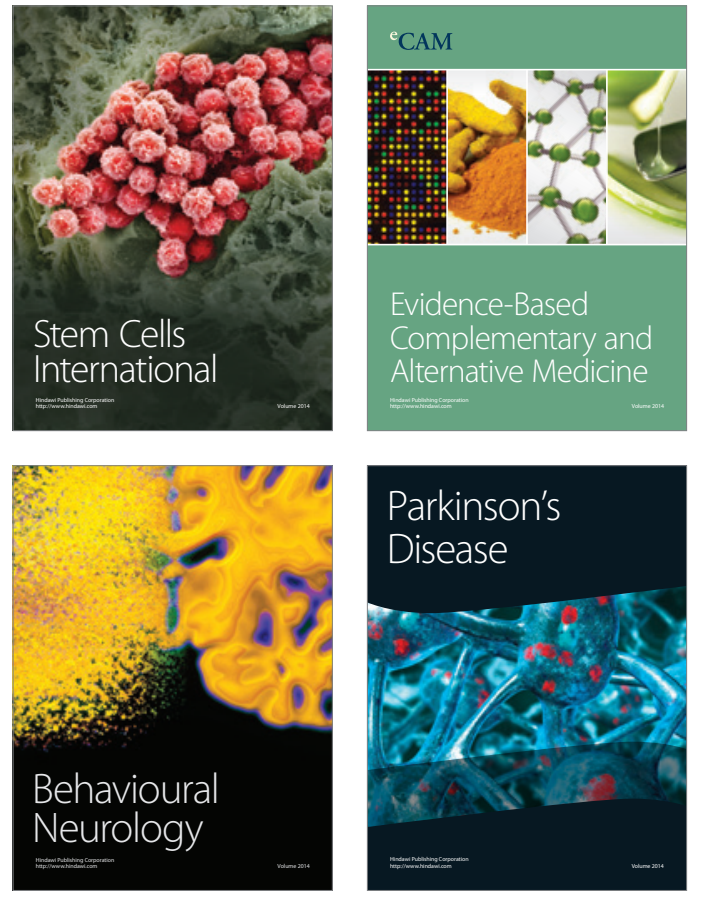
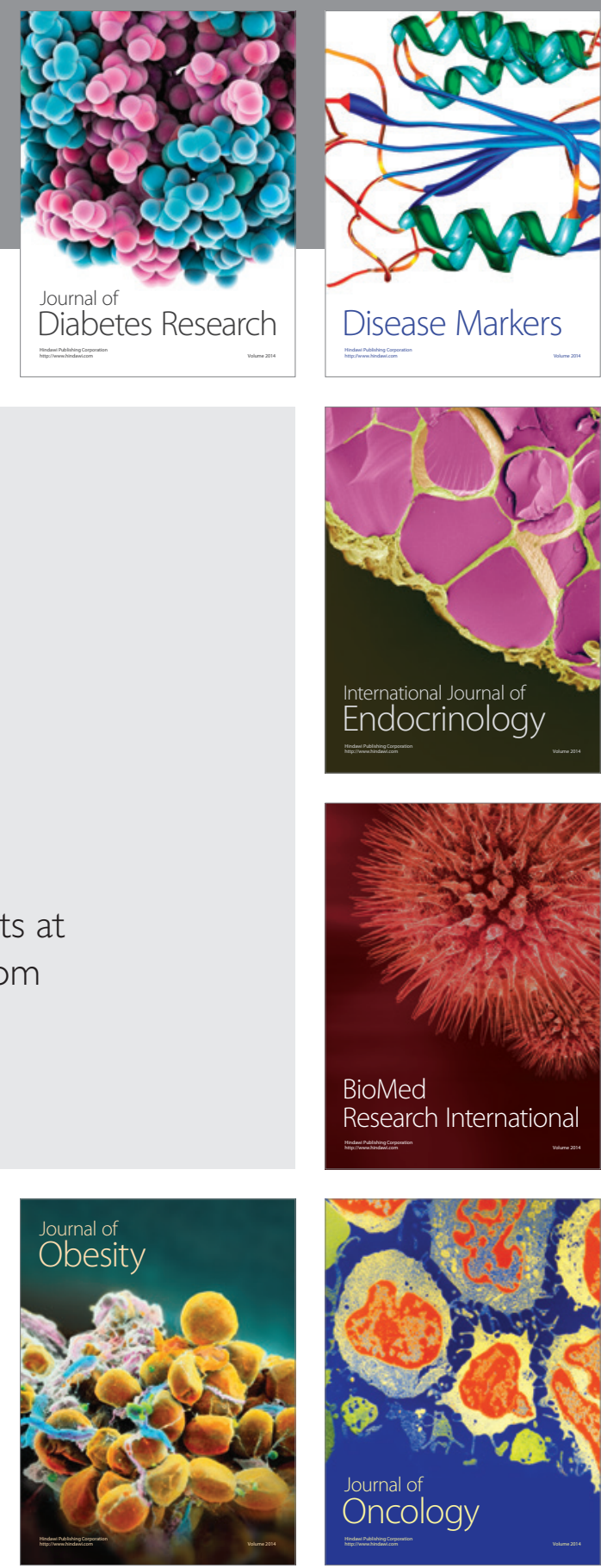

Disease Markers
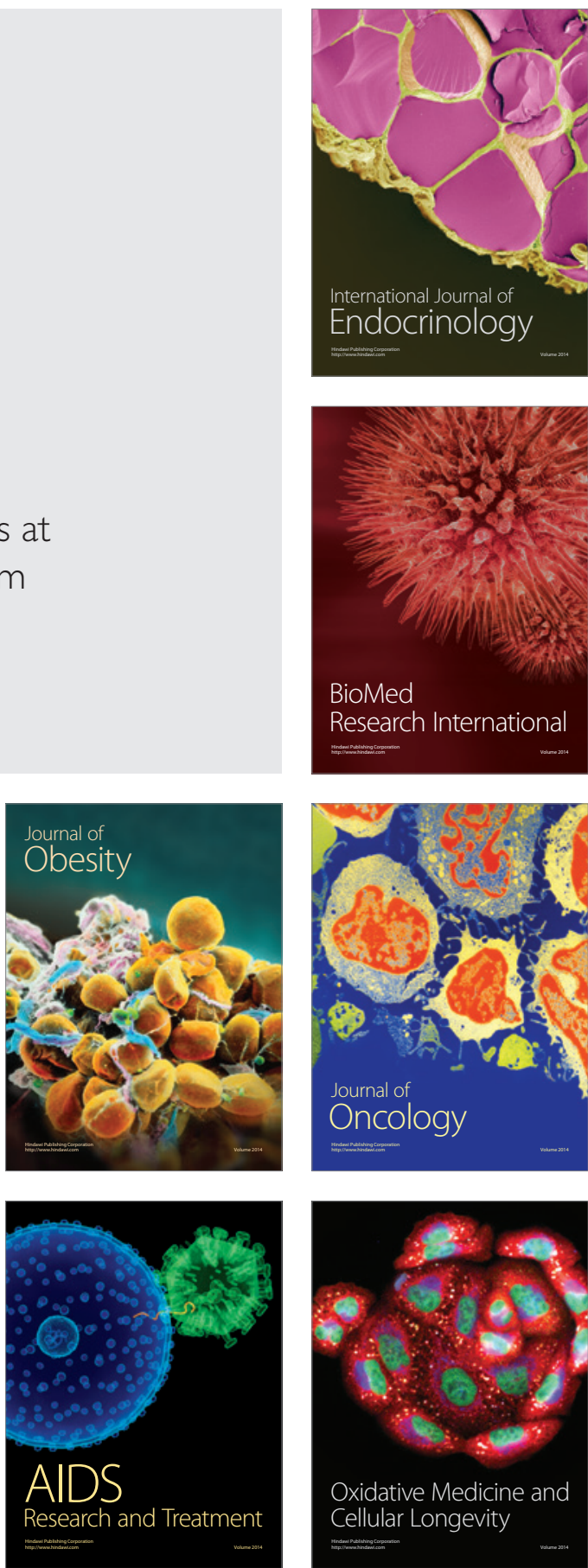